\title{
On the Equiconvergence of the Fourier Series and Integral of Distributions
}

\author{
A. A. Rakhimov \\ Department of Science in Engineering, International Islamic University Malaysia (IIUM), Kuala Lumpur, Malaysia \\ Email: abdumalik@iium.edu.my
}

Received 14 September 2015; accepted 13 November 2015; published 16 November 2015

Copyright (C) 2015 by author and Scientific Research Publishing Inc.

This work is licensed under the Creative Commons Attribution International License (CC BY). http://creativecommons.org/licenses/by/4.0/

c) (i) Open Access

\section{Abstract \\ We prove equiconvergence of the Bochner-Riesz means of the Fourier series and integral of dis- tributions with compact support from the Liouville spaces.}

\section{Keywords}

\section{Bochner-Riesz Means, Fourier Series, Fourier Integrals, Distributions, Equiconvergence}

\section{Introduction}

Convergence of the Fourier series and integral of integrable functions of one variable at certain point depends only from the values of the function in the small neighbourhood of this point (localizations principles). Moreover, the difference of the partial sums of the Fourier series and integral of a function uniformly converge to zero, which means both expansions converge or diverge at the same time (equiconvergence).

In $N$-dimensional case, $N>1$, localization principles, as well as equiconvergence, for the Fourier series and integral is not valid by the Pringsheim convergence [1]. In [2] it is given a review of recent results on equiconvergence of expansions in multiple trigonometric Fourier series and integral in the case of summation over rectangles. In [3] the problem of equiconvergence for expansions in a triple trigonometric Fourier series and a Fourier integral of continuous functions with a certain modulus of continuity in the case of a lacunary sequence of partial sums is studied.

In [4] equiconvergence of the Fourier interals and expansions associated with a Schrodinger operator is studied. In [5] the author obtained sufficient conditions on the potential under which uniform equiconvergence holds for the expansion of a integrable function in the system of eigenfunctions and associated functions of corresponding Sturm-Liouville operator and its Fourier sine series expansion (in [6] potential is a distribution). In [7] a comparison theorem on equiconvergence of the Fourier Jacobi series with certain trigonometric Fourier series is proved. 
In this paper we study equiconvergence of the Fourier series and integral of the linear continuous functionals (distributions) in the case of spherical summation. Localiation of spectral expansions of distributions for the first time was studied by Sh.A. Alimov [8]. Further results in [8] expanded to the more general spectral expansions in [9]-[14].

\section{Preliminaries}

Let $E\left(T^{N}\right)$ be the space of infinitely differentiable functions $\phi: T^{N} \rightarrow C$, with the locally convex topology produced from the system of the semi-norms

$$
P_{K, \gamma}(\phi)=\sup _{x \in K}\left|D^{\gamma} \phi(x)\right|,
$$

where $K$ is a compact subset of $T^{N}=(-\pi, \pi)^{N}, \gamma=\left(\gamma_{1}, \gamma_{2}, \cdots, \gamma_{N}\right), \quad \gamma_{j}(j=1,2, \cdots, N)$ is a non negative integer number, $|\gamma|=\gamma_{1}+\gamma_{2}+\cdots+\gamma_{N}$. and $D^{\gamma}=D_{1}^{\gamma_{1}} D_{2}^{\gamma_{2}} \cdots D_{N}^{\gamma_{N}}, \quad D_{j}=\frac{1}{i} \frac{\partial}{\partial x_{j}}, j=1,2, \cdots, N$.

Recall $E^{\prime}\left(T^{N}\right)$ the space of distributions on $E\left(T^{N}\right)$, i.e. the space of all continuous linear functionals on $E\left(T^{N}\right)$. In fact any element $f \in E^{\prime}\left(T^{N}\right)$ has a compact support in $T^{N}$ and can be represented as the weakly convergent Fourier series

$$
f=(2 \pi)^{-\frac{N}{2}} \sum_{n \in Z^{N}} f_{n} \exp (i n x),
$$

where its Fourier coefficients $f_{n}$ defined as the value of $f$ on the test function on $(2 \pi)^{-\frac{N}{2}} \exp (-i n x), \quad x \in T^{N}$ and $Z^{N}$ is the set of all $N$-tuples with integer coordinates.

The Riesz means of order $s, \quad s \geq 0$, of the spherical partial sums of the Fourier series (1) define by

$$
\sigma_{\lambda}^{s} f(x)=(2 \pi)^{\frac{-N}{2}} \sum_{|n|^{2}<\lambda}\left(1-\frac{|n|^{2}}{\lambda}\right)^{s} f_{n} \exp (\text { inx }) .
$$

Now, let us extend $f$ from $T^{N}$ to $R^{N}$ by zero and leave the same notation for $f$. Then recall the BochnerRiesz means of order $s$ of the Fourier integral of $f$

$$
R_{\lambda}^{s} f(x)=(2 \pi)^{\frac{-N}{2}} \int_{|y|^{2}<\lambda}\left(1-\frac{|y|^{2}}{\lambda}\right)^{s} \hat{f}(y) \exp (i y \cdot x) \mathrm{d} y,
$$

where $\hat{f}(y)=\left\langle f,(2 \pi)^{-\frac{N}{2}} \exp (-i y \xi)\right\rangle$ is the Fourier transformation of the distribution $f$ evaluated as its action on the test function $(2 \pi)^{-\frac{N}{2}} \exp (-i y \xi)$ with respect to the variable $\xi$.

In this paper we shall be studying a relation between expansions (2) and (3) for some values of the summation index $s$ depending on the power of singularity of $f$. In fact we will prove uniform equiconvergence of the Riesz means of the Fourier series and the Fourier integral expansion.

However, a behaviour of spherical means for the Fourier series and the Fourier integral expansion can be es sentially different. The first results on the different behaviour of the Riesz means of critical index $s=\frac{N-1}{2}$ of the Fourier integral and the Fourier series in $L_{1}$ found by S. Bochner [15], where it is proved that the localization of the means (3) holds and for the means (2) the localization fails. In the same paper [15] it is proved validity of localization principle in $L_{2}$ for both expansions in the critical index $s=\frac{N-1}{2}$. E. Stein [16] proved that if $s=\frac{N-1}{2}$ the localization principle for the means (2) remain valid in $L \log ^{+} L$ (consequently in $\left.L_{p}, p>1\right)$. 
In [17] B.M. Levitan reported the first result on the uniform equisummability of the Riesz means $\left(s \geq \frac{N-1}{2}\right)$ expansions associated with the Laplace operator. The Riesz equisummability below critical index $\frac{N-1}{2}$ studied by V.A. Il'in [18].

\section{Main Results}

For any real number $\ell$ by $L_{2}^{\ell}\left(T^{N}\right)$ denote the Liouville space of distributions

$$
L_{2}^{\ell}\left(T^{N}\right)=\left\{f \in E^{\prime}: \sum_{n \in Z^{N}}\left(1+|n|^{2}\right)^{\ell} f_{n}^{2}<\infty\right\} .
$$

Theorem 1 Let $\ell>0$ and $s=\frac{N-1}{2}+\ell$. Then for any $f \in L_{2}^{-\ell}\left(T^{N}\right)$

$$
\sigma_{\lambda}^{s} f(x)=R_{\lambda}^{s} f(x)+O(1)\|f\|_{-\ell}
$$

where $\|\cdot\|_{-\ell}$ a norm in $L_{2}^{-\ell}\left(T^{N}\right)$ :

$$
\|f\|_{-\ell}=(2 \pi)^{-\frac{N}{2}} \sqrt{\sum_{n \in Z^{N}}\left(1+|n|^{2}\right)^{-\ell} f_{n}^{2}} .
$$

Note, if $s<\frac{N-1}{2}+\ell$, then for any $x_{0} \in T^{N}$ there exist a distribution from $L_{2}^{-\ell}\left(T^{N}\right)$ such that it is coincides with zero in some neighbourhood of $x_{0}$ and the means $R_{\lambda}^{s} f\left(x_{0}\right)$ (the same for the means $\sigma_{\lambda}^{s} f\left(x_{0}\right)$ diverges [8]. Thus, formula (4) provides precise result on the uniform equiconvergence of the Riesz means of the Fourier Integral and Series.

The illustration of the domains of convergence in the Theorem 1 given in Figure 1 below and equiconver gence summation domain for the Dirac delta function $\delta \in L_{2}^{\ell}\left(T^{N}\right), \ell>\frac{N}{2}$ given in Figure 2.

\section{Estimation of the Direchlet Kernel}

Let $D_{\lambda}^{s}(x)$ be the Riesz means of the partial sums of the Fourier series of the Dirac delta function, which is well known as the Direchlet kernel:

$$
D_{\lambda}^{s}(x)=(2 \pi)^{-N} \sum_{|n|^{2}<\lambda}\left(1-\frac{|n|^{2}}{\lambda}\right)^{s} \exp (\text { inx }) .
$$

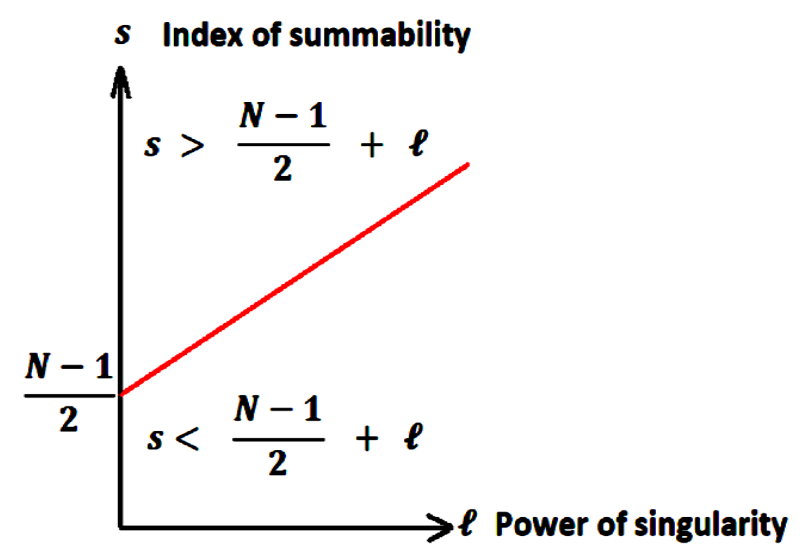

Figure 1. Localization of the Fourier Integral and Series. 


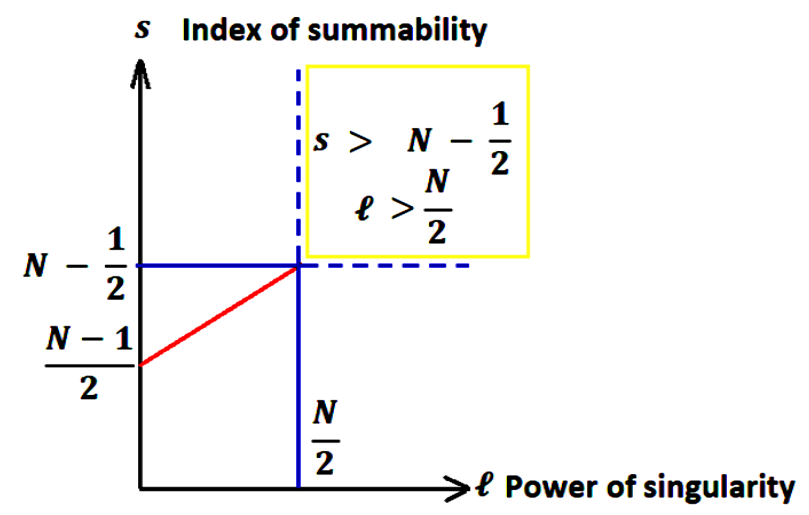

Figure 2. Localization domain for the Delta function.

Then for any distribution $f \in E^{\prime}$ Formula (2) can be expressed as

$$
E_{\lambda}^{s} f(x)=\left\langle f, D_{\lambda}^{s}(x-y)\right\rangle \text {, }
$$

where $f$ is acting to the test function $D_{\lambda}^{s}(x-y)$ by the variable $y$.

Similarly, for the Fourier integral (3) we write

$$
R_{\lambda}^{s} f(x)=\left\langle f, \Theta_{\lambda}^{s}(x-y)\right\rangle,
$$

where $\Theta_{\lambda}^{s}(x)$ the Bochner-Riesz means of the Fourier integral of the Dirac delta function:

$$
\Theta_{\lambda}^{s}(x)=(2 \pi)^{-N} \int_{|y|^{2}<\lambda}\left(1-\frac{|y|^{2}}{\lambda}\right)^{s} \mathrm{e}^{(i \mathrm{y} \cdot x)} \mathrm{d} y=(2 \pi)^{-N} 2^{s} \Gamma(s+1) \frac{\lambda^{\frac{N}{2}-s} J_{\frac{N}{2}+s}(\lambda|x|)}{|x|^{\frac{N}{2}+s}},
$$

Lemma 1 Let $\ell>0, s=\frac{N-1}{2}+\ell$ and $\hat{\Theta}_{\lambda}^{s}(\xi)$ be the Fourier transformation of the Riesz-Bochner kernel (8). Then

$$
\begin{gathered}
\left|\hat{\Theta}_{\lambda}^{s}(\xi)\right| \leq \operatorname{const}(1+|\xi|)^{-N-\ell} \\
\left|\Theta_{\lambda}^{s}(x)\right| \leq \operatorname{const}(1+|x|)^{-N-\ell}
\end{gathered}
$$

Proof. From the definition of the kernel $\Theta_{\lambda}^{s}(x)$ obviously obtain

$$
\hat{\Theta}_{\lambda}^{s}(\xi)= \begin{cases}\left(1-\frac{|\xi|^{2}}{\lambda}\right)^{s}, & \text { if }|\xi|^{2} \leq \lambda, \\ 0, & \text { otherwise. }\end{cases}
$$

Then estimate (9) immediately follows from (11). The estimate (10) follows from (8) and the estimate for the Bessel functions:

$$
\left|J_{v}(r)\right| \leq \text { const } \frac{1}{\sqrt{r}}, \quad r>1
$$

Lemma 1 proved.

Note, that if a function $g(x)$ and its Fourier transformation $\hat{g}(\xi)$ satisfy the estimates (9) and (10), then the Poisson formula for summation is valid:

$$
\sum_{n \in Z^{N}} g(x+2 \pi n)=(2 \pi)^{-\frac{N}{2}} \sum_{n \in Z^{N}} \hat{g}(n) \exp i n x
$$


Thus from Lemma 1 applying (12) for the function $g(x)=\Theta_{\lambda}^{s}(x)$ obtain

$$
\sum_{n \in Z^{N}} \Theta_{\lambda}^{s}(x+2 \pi n)=(2 \pi)^{-\frac{N}{2}} \sum_{n \in Z^{N}} \hat{\Theta}_{\lambda}^{s}(n) \exp i n x .
$$

Then from (5) and (11) we have

$$
D_{\lambda}^{s}(x)=\sum_{n \in Z^{N}} \Theta_{\lambda}^{s}(x+2 \pi n) .
$$

In the sum of right hand side in (13) by separation term $n=0$ obtain

$$
D_{\lambda}^{s}(x)=\Theta_{\lambda}^{s}(x)+\Theta_{*, \lambda}^{s}(x),
$$

where $\Theta_{*, \lambda}^{s}(x)$ defined as

$$
\Theta_{*, \lambda}^{s}(x)=\sum_{n \in Z^{N}, n \neq 0} \Theta_{\lambda}^{s}(x+2 \pi n) .
$$

Then from Lemma 1 immediately follows:

Lemma 2 Let $\ell>0, s=\frac{N-1}{2}+\ell$. Then uniformly in any compact set $K \subset T^{N}$

$$
\left|\Theta_{*, \lambda}^{s}(x)\right|=O\left(\lambda^{-\frac{\ell}{4}}\right)
$$

\section{Proof of the Theorem 1}

From the Formula (15) obtain

$$
\sigma_{\lambda}^{s} f(x)-R_{\lambda}^{s} f(x)=\left\langle f, \Theta_{*, \lambda}^{s}(x-y)\right\rangle .
$$

Then the statement of the Theorem 1 follows from the lemma below and equality (17):

Lemma 3 Let $s=\frac{N-1}{2}+\ell, \ell>0, \quad f \in L_{2}^{-\ell}\left(T^{N}\right) \cap E^{\prime}\left(T^{N}\right)$ and let suppf $\subset \Omega \subset \subset T^{N}$.

Then

$$
\left\langle f, \Theta_{*, \lambda}^{s}(x-y)\right\rangle=O(1)\|f\|_{-\ell}
$$

uniformly in any compact set $K \subset T^{N} \backslash \bar{\Omega}$.

Proof. For any proper domain $\Omega_{0} \subset \subset \Omega$

$$
\left|\left\langle f, \Theta_{*, \lambda}^{s}(x-y)\right\rangle\right| \leq\|f\|_{-\ell}\left\|\Theta_{*, \lambda}^{s}(x-y)\right\|_{\ell, 0}
$$

where $\|\cdot\|_{\ell, 0}$ means a norm in the space $L_{2}^{\ell}\left(\Omega_{0}\right)$ taken with respect to the variable $y \in \Omega_{0}$.

Note if $|x-y|>c$, then [19]

$$
\left\|\left\langle\Theta_{*, \lambda}^{s}(x-y)\right\rangle\right\|_{0}=O\left(\lambda^{\frac{-\ell}{4}}\right),
$$

where $\|\cdot\|_{0}$ means a norm in $L_{2}\left(\Omega_{0}\right)$.

Then the statement of the Lemma 4 follows from (19) and

$$
\left\|\left\langle\Theta_{*, \lambda}^{s}(x-y)\right\rangle\right\|_{\ell, 0}=O\left(\lambda^{\frac{\ell}{4}}\right)\left\|\Theta_{*, \lambda}^{s}(x-y)\right\|_{0},
$$

\section{Conclusion}

Equiconvergence of the Fourier series and integral of distributions depends on singularity of the distribution and power of regularisation as found in the main theorem. Obtained in Theorem 1 a relation for the singularity and 
summability index is accurate. However, to prove sharp result for the Reisz means below critical index for the smooth functions meets with some difficulties. This circumstance appears due to not applicability of the Poisson formula of summation.

\section{Acknowledgements}

Ongoing research on the topics of the paper supported by IIUM FRGS 141420383.

\section{References}

[1] Alimov, Sh.A., Il'in, V.A. and Nikishin, E.M. (1977) Problems of Convergence of Multiple Trigonometric Series and Spectral Decompositions. Russian Mathematical Surveys, 32, 115-139.

[2] Bloshanskii, I.L. and Grafov, D.A. (2014) Equiconvergence of Expansions in Multiple Trigonometric Fourier Series and Fourier Integral with Jk-lacunary Sequences of Rectangular Partial Sums. Acta et commentationes Universittatis Tartuensis de Mathematica, 18, 69-80.

[3] Grafov, D.A. (2015) Equiconvergence of Expansions into Triple Trigonometric Series and Fourier Integral for Continuous Functions with a Certain Modulus of Continuity. Moscow University Mathematics Bulletin, 70, $24-32$. http://dx.doi.org/10.3103/S0027132215010052

[4] Denisov, S.A. (1998) Equiconvergence of a Spectral Expansion Corresponding to a Schrodinger Operator with Summable Potential, with Fourier Integral. Differential Equations, 34, 1043-1048.

[5] Sadovnichaya, V. (2010) Equiconvergence Theorems for Sturm-Lioville Operators with Singular Potentials (Rate of Equiconvergence in $W_{2}^{\theta}$-Norm). Eurasian Mathematical Journal, 1, 137-146.

[6] Sadovnichaya, V. (2010) Equiconvergence of Eigenfunction Expansions for Sturm-Liouville Operators with a Distributional Potential. Sbornik: Mathematics, 201, 61-76.

[7] Marcokova, M. (1995) Equiconvergence of Two Fourier Series. Journal of Approximation Theory, 80, 151-163. http://dx.doi.org/10.1006/jath.1995.1012

[8] Alimov, Sh.A. (1993) On the Spectral Decompositions of Distributions. Doklady Mathematics, 331, 661-662.

[9] Alimov, Sh.A. and Rakhimov, A.A. (1996) Localization of Spectral Expansions of Distributions. Difference Equations, 32, 798-802.

[10] Alimov, Sh.A. and Rakhimov, A.A. (1997) Localization of Spectral Expansions of Distributions in a Closed Domain. Difference Equations, 33, 80-82.

[11] Rakhimov, A.A. (2000) On the Localization of Multiple Trigonometric Series of Distributions. Dokl. Math., 62, 163-165. (translation from Dokl. Akad. Nauk, Ross. Akad. Nauk, 374, 20-22).

[12] Rakhimov, A.A. (1996) Localization Conditions for Spectral Decompositions Related to Elliptic Operators from Class $A_{\mathrm{r}}$. Mathematical Notes, 59, 298-302. (translation from Mat. Zametki, 59, 421-427).

[13] Rakhimov, A., Ahmedov, A. and Zainuddin, H. (2012) On the Spectral Expansions of Distributions Connected with Schrodinger Operator. Applied Mathematics Letters, 25, 921-924.

[14] Rakhimov, A.A. (1996) Spectral Decompositions of Distributions from Negative Sobolev Classes. Difference Equations, 32, 1011-1013. https://zbmath.org/?q=an:0890.47031

[15] Bochner, S. (1936) Summation of Multiple Fourier Series by Spherical Means. Transactions of the American Mathematical Society, 40, 175-207. http://dx.doi.org/10.1090/S0002-9947-1936-1501870-1

[16] Stein, E.M. (1958) Localization and Summability of Multiple Fourier Series. Acta Mathematica, 100, 93-147. http://dx.doi.org/10.1007/BF02559603

[17] Levitan, B.M. (1954) On the Eigenfunction Expansions of the Laplace Operator. Matematicheskii Sbornik, 35, 267316.

[18] Il’in, V.A. (1995) Spectral Theory of Differential Operators: Self-Adjoint Differential Operators. Consultants Bureau, New York.

[19] Bastis, A.Y. (1983) On the Asymptotic of the Riesz-Bochner Kernel. Analysis Mathematica, 9, 247-258. http://dx.doi.org/10.1007/BF01910305 